\title{
THE
}

\section{A Comparison of a Single Receiver and a Multi-Receiver Techniques to Mitigate Partial Band Interference}

James McGee

Steven Schoenecker

Josko Catipovic

Peter F. Swaszek

University of Rhode Island, swaszek@uri.edu

Follow this and additional works at: https://digitalcommons.uri.edu/ele_facpubs

The University of Rhode Island Faculty have made this article openly available.

Please let us know how Open Access to this research benefits you.

This is a pre-publication author manuscript of the final, published article.

Terms of Use

This article is made available under the terms and conditions applicable towards Open Access Policy Articles, as set forth in our Terms of Use.

\section{Citation/Publisher Attribution}

McGee, J., Schoenecker, S., Catipovic, J., \& Swaszek, P. (2014). A Comparison of a Single Receiver and a Multi-Receiver Techniques to Mitigate Partial Band Interference." Proceedings of the International Conference on Underwater Networks \& Systems (WUWNET '14), pp. 1-5. doi: 10.1145/2671490.2674563 Available at: http://dx.doi.org/10.1145/2671490.2674563

This Conference Proceeding is brought to you for free and open access by the Department of Electrical, Computer, and Biomedical Engineering at DigitalCommons@URI. It has been accepted for inclusion in Department of Electrical, Computer, and Biomedical Engineering Faculty Publications by an authorized administrator of DigitalCommons@URI.For more information, please contact digitalcommons-group@uri.edu. 


\title{
A Comparison of a Single Receiver and a Multi-Receiver Techniques to Mitigate Partial Band Interference
}

\author{
James McGee, Josko Catipovic and Steven Schoenecker \\ Naval Undersea Warfare Center \\ Newport, RI 02841 \\ Email: james.a.mcgee@navy.mil \\ Email: josko.catipovic@navy.mil \\ Email: steven.schoenecker@navy.mil
}

\author{
Peter Swaszek \\ Department of Electrical, Computer and Biomedical Engineering \\ University of Rhode Island \\ Kingston, RI 02881 \\ Email: swaszek@ele.uri.edu
}

\begin{abstract}
Many acoustic channels suffer from interference which is neither narrowband nor impulsive. This relatively long duration partial band interference can be particularly detrimental to system performance. We survey recent work in interference mitigation as background motivation to develop a spatial diversity receiver for use in underwater networks and compare this novel multi-receiver interference mitigation strategy with a recently developed single receiver interference mitigation algorithm using experimental data collected from the underwater acoustic network at the Atlantic Underwater Test and Evaluation Center. The network consists of multiple distributed cabled hydrophones that receive data transmitted over a time-varying multipath channel in the presence of partial band interference produced by interfering active sonar signals. In operational networks, many "dropped" messages are lost due to partial band interference which corrupts different portions of the received signal depending on the relative position of the interferers, information source and receivers due to the slow speed of propagation.
\end{abstract}

\section{INTRODUCTION}

The U. S. Navy operates an undersea cellular network in the Tongue of the Ocean [1]. Known as the Atlantic Undersea Test and Evaluation Center (AUTEC), it consists of 96 acoustic sensors placed over a 60 by 30 kilometer square area and is shown in Fig. 1. As currently configured, approximately 97 percent of transmitted messages are successfully decoded; of the remaining three percent, many are corrupted by acoustic interference arising from active acoustic emissions. For example, Fig. 2 shows the impact of interference on a received data packet. In pane (a), the data packet was received without interference and successfully decoded in contrast to pane (b) where interfering signals are clearly evident and the message was lost. Years of extensive observations of activities in the vicinity of the network demonstrate that the widely separated hydrophones suffer from partial-band interference emanating from multiple spatially separated sources. The nature of this interference is different from the impulsive or narrowband interference typically encountered in other applications. Furthermore, unlike RF communications and acoustic array processing applications where interference is highly correlated in time among the various receivers, in the acoustic network, interference affects different portions of the received signals due to the wide separation of the receivers and the low speed of propagation. The degradation in the received signal is highly variable, depending on the relative position of the interfering signals, information source and receivers as well as the channel conditions. Successful steps to mitigate interference using a single receiver have been reported in [2], and the potential of using multiple receivers to mitigate interference was demonstrated in [3]. The motivation behind this work is to compare the potential benefits that each mitigation strategy provides.

Interference mitigation has a long history in RF communications, but the interference is typically impulsive or narrowband [4]. Partial band interference is not addressed [2]. The interference mitigation techniques typically exploit the short time or limited frequency span of the interfering signal. Examples of impulsive noise suppression techniques for multi-carrier modulation may be found in [5]-[11], while [12]-[21] address narrowband interference mitigation. Early approaches tended to separate channel estimation and interference detection, while more recent work has focused on jointly estimating the channel and mitigating interference. Joint approaches may work iteratively such as in [6] or by expanding the states of the decoding algorithm as in [16] and [18]. A message-passing approach to jointly estimating the channel and mitigating strong co-channel interference of similar form as the desired signal was proposed in [22]. Two blind algorithms to mitigate multiple interferers were proposed in [23]. Joint approaches provide better performance at the cost of additional computational complexity. Limitations on system performance may be found in [24] for OFDM systems subject to impulsive noise and for multicarrier and single carrier quadrature amplitude 


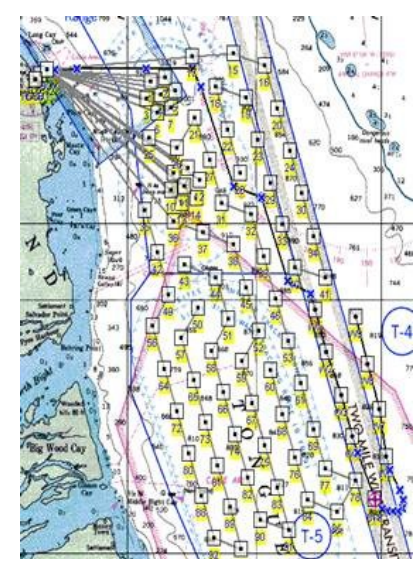

Figure 1. The AUTEC acoustic network.

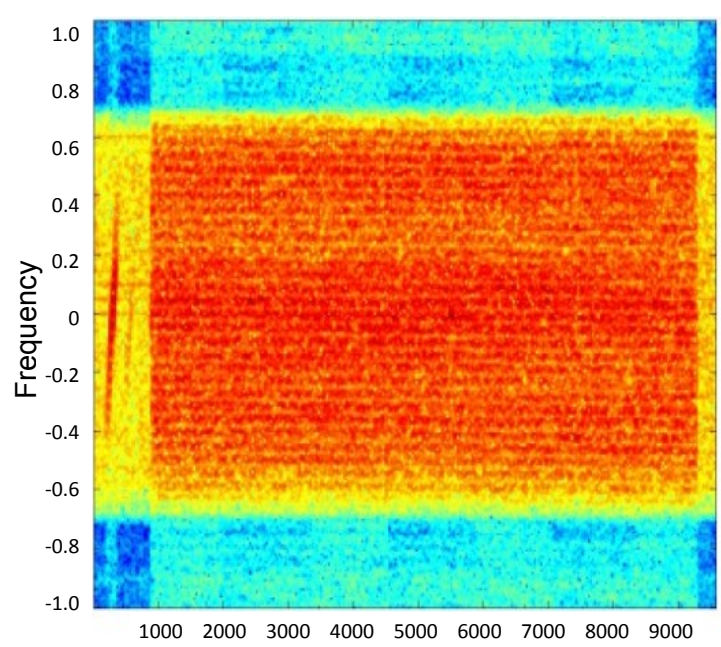

(a)

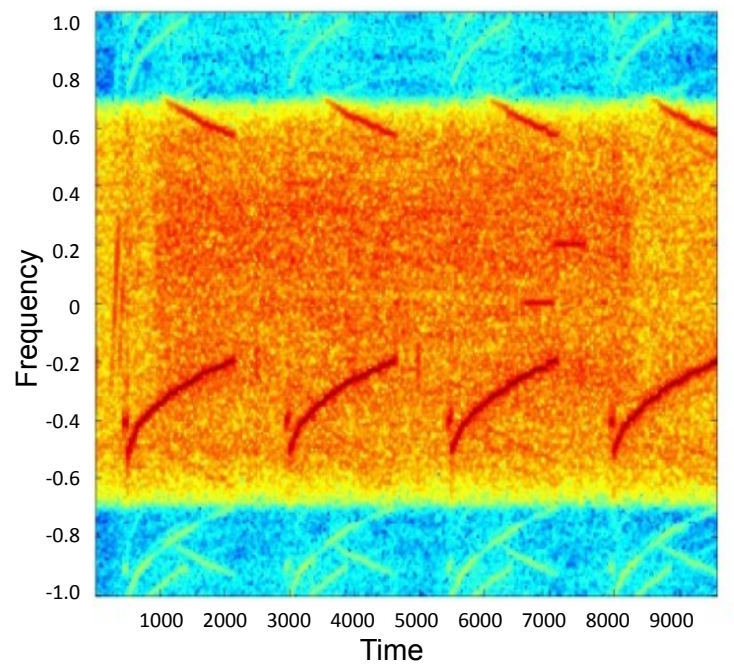

(b)

Figure 2. Clean packet reception (a) and packet corrupted by acoustic interference (b).

modulation (QAM) systems in [25]. The capability of low density parity check (LDPC) and turbo coding to mitigate burst errors is discussed in [26].

Observations from past field experiments indicate that significant improvement in the reliability of message reception can be realized by mitigating interference. Discussion of the interference typical in the underwater environment is available in [27] and [28].

The work in [2] developed a single receiver parameterized interference cancellation (PIC) algorithm to mitigate partial band interference through a representation of the interference based on a rough estimate of its time duration and bandwidth. The PIC algorithm explicitly cancelled the interference without information loss or performance degradation caused by approximations. In [3] we developed a multi-receiver algorithm that relied on the spatial diversity inherent in an acoustic network to facilitate the reconstruction of the transmitted waveform from the clean portions of the received signals. Our algorithm also relied on the known time duration and bandwidth of the interference to perform mitigation. While both approaches rely on prior knowledge of the interference band and time duration, the techniques differ in the use of the knowledge. In [2] prior knowledge aids in the estimation of the interference parameters and facilitates coherent cancellation where as in [3] prior knowledge facilitates the detection and excision of portions of the received signal that suffer from interference. For both approaches, knowledge of the interference parameters does not need to be particularly precise and can be determined in situ. The experiment reported here used Linear Frequency Modulated (LFM) signals as interference. However, both approaches are applicable to any time and band limited signal such as Hyperbolic Frequency Modulated (HFM) waveforms or marine mammal vocalizations.

\section{A. Parameterized Interference Cancellation}

The work in [2] developed an iterative single receiver algorithm to mitigate partial band interference of known time duration, $T_{I}$, and interference bandwidth, $B_{I}$. Since the interference is time and band limited, it can be represented by a Fourier-series. 


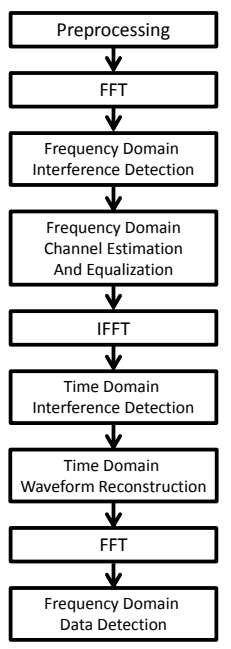

Figure 3. OFDM waveform reconstruction process.

The algorithm employs an iterative approach to estimate the $N_{I}=\left\lceil B_{I} T_{I}\right\rceil$ complex coefficients as well as the time delay (with respect to the start of the OFDM block) of the interference. On each iteration, the estimates of the interference and detected symbols from the previous iteration are used to construct a Generalized Likelihood Ratio Test (GLRT) to see if interference is still present. If residual interference is detected, its model parameters are calculated with a maximum-likelihood approach. The modeled interference is than subtracted out, and the channel is equalized and symbol estimation is again attempted. This is repeated until the parity checks are satisfied or some maximum preset iteration limit is reached. 


\begin{tabular}{|l|l|l|}
\hline \multicolumn{2}{|c|}{ OFDM Parameter } & Value \\
\hline Center frequency & $f_{c}$ & $11 \mathrm{kHz}$ \\
\hline Bandwidth & $B$ & $6 \mathrm{KHz}$ \\
\hline \# of subcarriers & $K$ & 1024 \\
\hline \# data subcarriers & $\left|\mathcal{S}_{D}\right|$ & 86 \\
\hline \# pilot subcarriers & $\left|\mathcal{S}_{P}\right|$ & 256 \\
\hline \# null subcarriers & $\left|\mathcal{S}_{N}\right|$ & 96 \\
\hline Symbol Duration & $T$ & $104.68 \mathrm{~ms}$ \\
\hline Symbol Constellation & & BPSK \\
\hline Subcarrier spacing & $\Delta f=1 / \mathrm{T}$ & $9.54 \mathrm{~Hz}$ \\
\hline Guard interval & $T_{g}$ & $24.6 \mathrm{~ms}$ \\
\hline Number of Guard Samples & $N_{g}$ & 240 \\
\hline Synthesized Interference Parameters & Value \\
\hline Center Frequency & $f_{c, I}$ & $11 \mathrm{kHz}$ \\
\hline Bandwidth & $B_{I}$ & $B / 2$ \\
\hline Duration & $T_{I}$ & $T / 3$ \\
\hline
\end{tabular}

Table 1. EXPERIMENT PARAMETERS.

\section{B. Spatial Diversity Reciever}

The spatial diversity receiver identifies portions of the signal suffering from interference on different receivers, removes these portions of the signal and then optimally combines the remaining clean portions of the signal. The process, illustrated Fig. 3, involves switching between the time and frequency domain because interference excision and waveform recombining must be done on equalized time-domain waveforms and all of the information for channel estimation is present in the frequency (or OFDM symbol) domain. Pre-processing synchronizes the received signals and adjusts for Doppler induced dilation or contraction. The signals are then transferred to the frequency domain for removal of any residual Doppler effect, channel equalization and testing for the presence of interference. The frequency domain interference detector compares the signal power on the null subcarriers inside and outside of the interference band to determine if interference is present. Signals having interference are transferred back to the time domain where knowledge of the interference time duration aids in determining which portion of the time domain waveform suffers from interference. Reconstruction of time domain waveform excises time orthogonal interference and averages across portions of the waveform suffering from only noise. If the interference is not time orthogonal (e.g. it occupies the same time bins on all receivers), averaging is performed. The synthesized signal is then transferred back to the frequency domain for data detection.

\section{EXPERIMENTAL RESULTS}

During May 2014, experiments were conducted at AUTEC to test the interference mitigation algorithms. A multichannel projector was configured for multichannel simultaneous transmission of both interfering and desired acoustic waveforms. The desired OFDM signal consisted of a channel probe, four data packets and a final channel probe while LFM waveforms of various time durations and bandwidths provided the interference. Multiple interfering and desired waveforms were simultaneously transmitted from AUTEC's acoustic transmitters and recorded on more than forty distributed ocean-bottom hydrophones. Examples of the received data packets are shown in Fig 4. The interfering LFM waveform is clearly evident in both spectrograms. In addition to the LFM interference, the third data block suffers interblock interference (IBI) as portions of the surface bounce arrival of the first data block coincides with the direct path arrival of the third data block.

Independently processing the third block from the channels shown in Fig 4 without interference suppression resulted in thirteen errors on channel 68 and eight errors on channel 76 where as applying the parameterized interference mitigation algorithm resulted in no errors. Processing the block using maximum ratio combining (MRC) resulted in three errors while applying spatial diversity reconstruction (SDR) resulted in no errors. The distance between these receivers is approximately 4,000 yards.

In order to test the performance of the algorithms at different interference and noise level, a data set of 1,500 synthesized received signals was created from the interference and noise sampled during the experiment. The pseudo-experimental interference was created by windowing the appropriate portions of the received direct path LFM signals and amplifying them to the appropriate interference level.. Similarly, noise from portions of the experiment when no signal or interference was present was amplified to the appropriate level to vary the signal to noise ratio. The amplified interference and noise was added to received OFDM blocks in which the symbols were all detected correctly prior to decoding. For testing of the SDR and MRC algorithms, the interference was time orthogonal on the two receivers. Table I-B provides details about the experiment.

The results of the processing the pseudo-experimental signals prior to decoding are shown in 5 for signal channel processing, MRC and SDR. A clairvoyant (shown in cyan diamonds) and non-clairvoyant (shown in blue circles) SDR algorithm were tested. The clairvoyant algorithm always excised the correct portion of the signal suffering interference whereas the non-clairvoyant algorithm had to detect the presence of interference as well as select the portion of the received signal to excise. At low SIRs (when the interference is large), there is a clear benefit to excising the interference for all values of SNR but more dramatic at higher SNRs. As the interference power approaches the noise power, the benefit of excising the interference diminishes and the loss from not averaging over the noise becomes apparent. This is demonstrated at higher SIRs in the results for the clairvoyant SDR receiver where applying the algorithm, even with perfect knowledge of the time window suffering interference, results in 

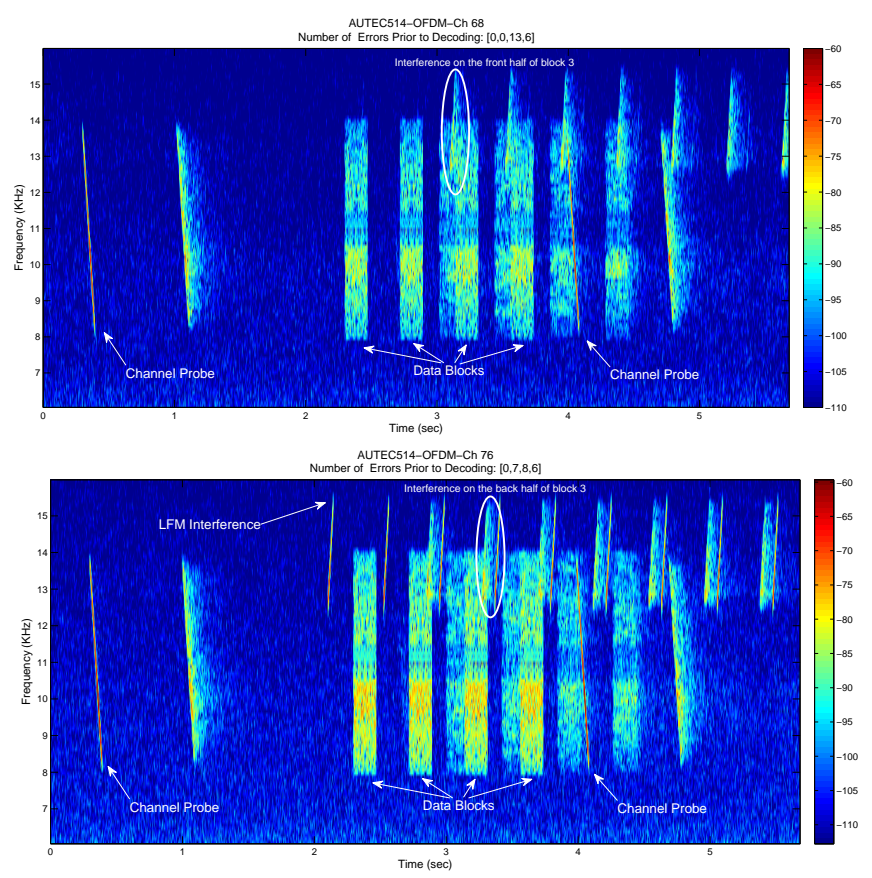

(a)

(b)

Figure 4. The OFDM signals received on channels 68 (panel a) and 76 (panel b) during an experiment at AUTEC in May 2014. Note that LFM interference corrupts for the beginning of block 3 of the data packet on channel 68 and the end of the same block on channel 76. The receivers are separated by approximately 4,000 yards.

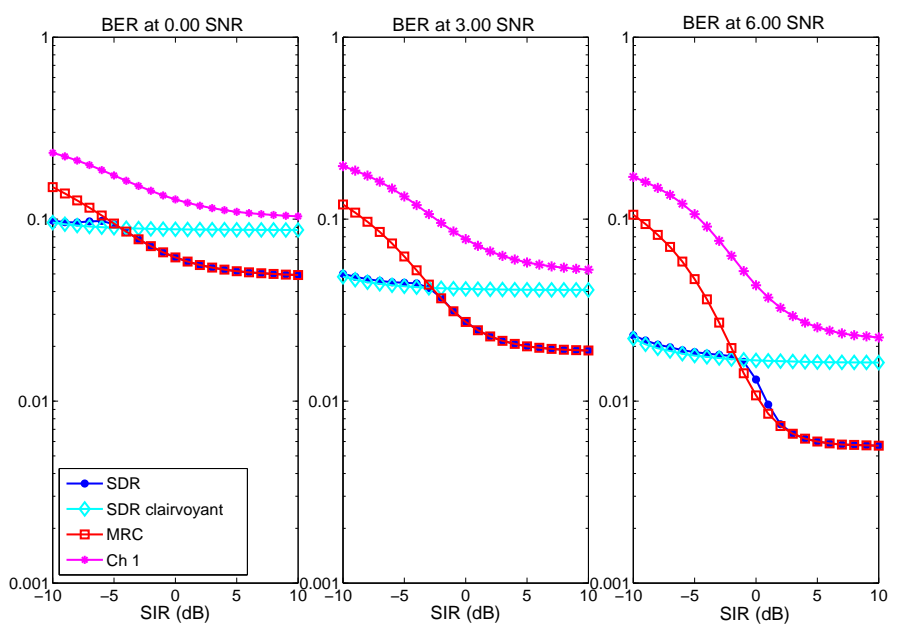

Figure 5. Bit error rate performance as a function of SIR for the different mitigation strategies.

degraded performance as compared to MRC . However, the interference detection threshold can be set to smoothly transition between the SDR and MRC combining strategies. By only declaring interference when it is sufficiently large, the SDR receiver without clairvoyance exhibits the same performance as MRC at high SIRs.

Fig. 6 shows the bit error rate after decoding for the various receivers. (The single channel uncoded bit error rate is also shown for comparison). The single channel PIC algorithm (green triangles) is effective at moderate SIR levels. This is the region of where Fig. 5 indicated the most effective strategy was to transition SDR to MRC. The PIC technique is ineffective at high SIRs; precisely the region where the SDR gains are most dramatic. Thus, the two approaches are complimentary and an effective multi-channel receiver strategy would be to adaptively utilize both techniques.

\section{SUMMARY}

Many acoustic channels suffer from interference which is neither narrowband nor impulsive. This relatively long duration partial band interference can be particularly detrimental to system performance. However, some parameters of the interference 


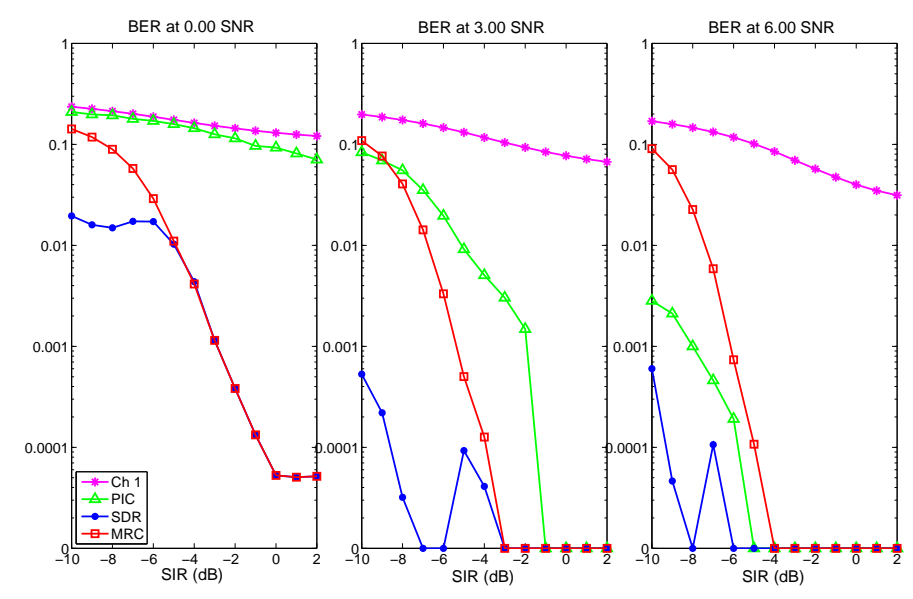

Figure 6. Coded bit error rate performance as a function of SIR for the different mitigation strategies.

are usually known or can be acquired and exploited to facilitate interference cancellation on a single receiver. For multiple receivers, the prior knowledge can be used to exploit the detection and excision of portions of the received signals suffering from interference so that they can be combined more effectively. While the single receiver technique relies on accurately estimating and subtracting the interference, the multi-receiver technique exploits the slow speed of sound propagation in water and the geographical extent of networks, to combine clean portions of the received signals. The experimental results from AUTEC demonstrate that both techniques are effective mitigation strategies. Parameterized interference cancellation is most effective at moderate SIRs whereas spatial diversity reconstruction is effective and realizes the most gain at high SIRs. The two approaches are complimentary and an effective multi-channel receiver strategy would be to adaptively utilize both techniques.

\section{REFERENCES}

[1] R. Hayford, D. Nagle, and J. Catipovic, "Undersea cellular network, Tongue of the Ocean Bahamas," in Proc. of the 4th ACM Workshop on Underwater Network (WuWNet), Berkerly, 2009.

[2] Z. Wang, S. Zhou, J. Catipovic, and P. Willett, "Parameterized cancellation of partial-band partial-block-duration interference for underwater acoustic OFDM," IEEE Transactions on Signal Processing, vol. 60, no. 4, pp. 1782-1795, 2012.

[3] J. McGee, J. Catipovic, and P. Swazsek, "Leveraging spatial diversity to mitigate partial band interference in undersea networks through waveform reconstruction," in to appear in Proc. of MTS/IEEE OCEANS Conf., 2014.

[4] J. Laster and J. Reed, "Interference rejection in digital wireless communications," IEEE Signal Process. Mag., vol. 14, no. 3, pp. $37-62$, 1997.

[5] S. Zhidkov, "Impulsive noise suppression in OFDM-based communication systems," IEEE Trans. Consum. Electron., vol. 49, no. 4, pp. 944-948, 2003.

[6] A. Mengi and A. Vinck, "Successive impulsive noise suppression in OFDM," in Proc. IEEE Int. Symp. on Power Line Commun. Appl., 2010.

[7] M. F.-G. García, J. Rojo-Álvarez, F. Alonso-Atienza, and M. Martínez-Ramón, "Support vector machines for robust channel estimation in OFDM," IEEE Signal Process. Lett., vol. 13, no. 7, pp. 397-400, 2006.

[8] X. Yu, P. Lin, Z. He, and W. Wu, "OFDM channel estimation with impulse noise cancellation,” in Proc. Int. Conf. on Wireless Commun., 2007, pp. 330-333.

[9] G. Caire, T. Al-Naffouri, and A. Narayanan, "Impulse noise cancellation in OFDM: An application of compressed sensing," in Proc. IEEE Int. Symp. Inf. Theory, 2008, pp. 1293-1297.

[10] K. Pelekanakis, H. Liu, and M. Chitre, "An algorithm for sparse underwater acoustic channel identification under symmetric alpha-stable noise," in Proc. of MTS/IEEE OCEANS Conf., 2011.

[11] J. Armstrong and H. A. Suraweera, "Decision directed impulse noise mitigation for OFDM in frequency selective fading channels," in Proc. GLOBALCOM, 2004, pp. 3536-3540.

[12] R. Nilsson, F. Sjoberg, and J. LeBlanc, "A rank-reduced LMMSE canceller for narrowband interference suppression in OFDM-based system," IEEE Trans. Commun., vol. 51, no. 12, pp. 2126-2140, 2003.

[13] D. Darsena, "Successive narrowband interference cancellation for OFDM systems," IEEE Commun. Lett., vol. 11, pp. 73-75, 2007.

[14] D. Darsena and F. Verde, "Successive NBI cancellation using soft decisions for OFDM systems," IEEE Signal Process. Lett., vol. 15, pp. 873-876, 2008.

[15] A. Jeremic, T. A. Thomas, and A. Nehorai, "OFDM channel estimation in the presence of interference," IEEE Trans. Signal Process., vol. 52, no. 12, pp. 3429-3439, 2004.

[16] M. Morelli and M. Moretti, "Channel estimation in OFDM systems with unknown interference," IEEE Trans. Wireless Commun., vol. 8, no. 10, pp. 5338-5347, 2009.

[17] _ , "Improved decoding of BICM-OFDM transmissions plagued by narrowband interference," IEEE Trans. Wireless Commun., vol. 10, no. 1, pp. 20-26, 2011.

[18] T. Li, W. H. Mow, V. Lau, M. Siu, R. Cheng, and R. Murch, "Robust joint interference detection and decoding for OFDM-based cognitive radio systems with unknown interference," IEEE J. Sel. Areas Commun., vol. 25, no. 3, pp. 566-575, 2007.

[19] A. Gomaa, K. M. Z. Islam, and N. Al-Dhahir, "Two novel compressive-sensing algorithms for NBI detection in OFDM systems," in Proc. Int. Conf. on Acoust., 2010, pp. 3294-3297. 
[20] T. Al-Naffouri, A. Quadeer, and G. Caire, "Impulsive noise estimation and cancellation in DSL using orthogonal clustering," in Proc. IEEE Int. Symp. Inf. Theory, 2011, pp. 2841-2845.

[21] K. Fazel, "Narrow-band interference rejection in orthogonal multicarrier spread-spectrum communications," in Third Annual International Conference on Universal Personal Communications, 1994, pp. 46-50.

[22] Y. Zhu, D. Guo, and M. L. Honig, "A message-passing approach for joint channel estimation, interference mitigation, and decoding," IEEE Trans. Wireless Commun., vol. 8, no. 12, pp. 6008-6018, 2009.

[23] F. He, X. Wang, T. Yang, B. Hu, and R. Liu, "Two blind algorithms of MMSE filter based on second-order statistics in MIMO systems with multiple interferences," in Wireless Communications and Signal Processing (WCSP), 2011.

[24] S. V. Zhidkov, "Performance analysis and optimization of OFDM receiver with blanking nonlinearity in impulsive noise environment," IEEE Trans. Vehicular Technology, vol. 55, no. 1, pp. 234-242, Jan. 2006Jan.

[25] M. Ghosh, "Analysis of the effect of impulse noise on multicarrier and single carrier QAM systems," IEEE Trans. Commun., vol. 44, no. 2, pp. 145-147, 1996.

[26] S. J. Johnson, Iterative Error Correction: Turbo, Low Density Parity-Check and Repeat Accumulate Codes. New South Wales: Cambridge University Press, 2010.

[27] J. Ward, M. Fitzpatrick, N. Dimarzio, D. Moretti, and R. Morrizzey, "New algorithm for open ocean mammal monitoring," in Proc. MTS/IEEE OCEANS Conf., 2000.

[28] J. Catipovic, "Robust acoustic communication with underwater vehicles in noisy and jammed shallow water environments," in OCEANS 1992: Mastering the Oceans Through Technology, 1992. 